\title{
TOTAL MASS AND LUMINOSITY OF STAR FORMING REGIONS IN THE LMC: PREDICTED INFRARED FLUXES
}

\author{
V. MISSOULIS, A. DAPERGOLAS AND E. KONTIZAS \\ Astronomical Institute, National Observatory of Athens \\ M. KONTIZAS \\ Department of Physics, National University of Athens \\ AND
}

S. OLIVER

Astrophysics Group, Blackett Laboratory, Department of Physics, Imperial College, London

We have investigated 4 Shapley constellations $(1996, A \& A, \mathbf{3 0 8}, 40)$ to define the associated stellar complexes and their properties. From observed star counts and recent spectral classification we derive an age estimate and the upper mass limit of individual stars in these regions.

Theoretical isochrones and the star counts allow us to find the total mass and luminosity in the optical band of each complex. For the stellar complex in Shapley I there are IRAS observations $(1991, A \& A, \mathbf{2 4 5}, 635)$ providing $12-100 \mu \mathrm{m}$ flux data for this region. From our observations in the optical bands, theoretical isochrones and the available infrared observations we have derived the ratios of $L_{\mathrm{IR}} / L_{\mathrm{B}}=80$ and $M / L_{\mathrm{IR}}=0.03$ with errors of a factor of 2 . The first ratio is much larger than in the majority of luminous infrared galaxies (IRG) and the second ratio is typical for an IRG. The temperature of the dust in Shapley I should be smaller than in an IRG since the stellar population in an IRG is younger than the age of $10^{8} \mathrm{yr}$ for Shapley I. Nevertheless the star formation in an IRG is taking place only in the central regions whereas in a star forming region it is taking place in the whole volume, but there is also a large number of old stars. So the ratio $M / L_{\mathrm{IR}}$ in both cases is affected equivalently by stars which do not heat the dust. However, the ratio $L_{\mathrm{IR}} / L_{\mathrm{B}}$ is affected strongly by stars which do not heat the dust in an IRG, but is affected only slightly in a star forming region.

Acknowledgements: One of us (V.Missoulis) is grateful to the LOC for financial support to attend the General Assembly. 